\title{
GROUPS WHICH ARE COGROUPS
}

\section{L. CURTIS AND J. DUGUNDJI}

It is the purpose of this note to give an elementary proof that $S^{0}, S^{1}, S^{3}$ are the only spheres which can be made into topological groups. This was proved by Samelson in [3]. He showed that a compact Lie group which is a homotopy sphere must have rank 1 , and then that a compact Lie group of rank 1 has dimension 1 or 3 . We get the first part easily by showing that a compact Lie group which is an $H$-cogroup (e.g., a suspension) must have rank 1 . The second part closely follows Samelson's proof. For basic facts about $H$-groups and $H$-cogroups the reader is referred to $\S \S 5$ and 6 of Chapter I of Spanier [5].

If $G$ is an $H$-group which is also an $H$-cogroup, then the set $[G, G]_{0}$ of pointed homotopy classes is an abelian group with its operation given equally by the $H$-cogroup structure on the domain $G$ or the $H$-group structure on the range $G[5$, page 44$]$. From this the following lemma is immediate.

LemMA 1. If $G$ is an $H$-group which is also an $H$-cogroup, $i: G \rightarrow G$ is the identity map and $\psi: G \rightarrow G$ is the squaring map $x \mapsto x^{2}$, then

$$
[\psi]=2[i] \in[G, G]_{0} .
$$

Next we need a special case of a result due to Hopf [2].

Lemma 2. If $G$ is a compact connected Lie group of rank $r$ and $\psi$ is the squaring map, then degree $\psi=2^{r}$.

PROOF. We will count the algebraic number of times a point $y \in G$ is covered. We may assume $y$ is regular (i.e., lies in a unique maximal torus) because the set of singular (= not regular) points forms a set of dimension less than the dimension of $G$ (indeed, the dimension is at most $\operatorname{dim} G-3$; see [4, p. 19]).

Let $T$ be the unique maximal torus containing $y$. Since $\operatorname{dim} T=r$ we easily find that $y$ has exactly $2^{r}$ square roots in $T$. If $z$ is any other square root of $y$, then $z$ lies in some other maximal torus $T^{\prime} \neq T$ (since maximal tori cover $G$ ). But then $y=z^{2} \in T^{\prime}$, contradicting the regularity of $y$. Thus there are exactly $2^{r}$ points $x \in G$ with $\psi(x)=y$.

We assert that all such $x$ cover $y$ with the same orientation. Given $x$ with $x^{2}=y$, let $U$ be a small open neighborhood of $x$ containing no other roots of $y$ and such that $U$ is star-shaped relative to $x$. Then

Received by the editors September 13, 1968. 


$$
\psi: U \rightarrow G
$$

is a diffeomorphism (if $U$ is small enough). Furthermore, if $T_{x}: U \rightarrow G$ is translation by $x$ it is easy to get an isotopy between $\psi$ and $T_{x}$ on $U$. Since $T_{x}$ preserves orientation, so does $\psi$. Hence a neighborhood of $y$ is covered $2^{r}$ times with the same orientation. Hence the degree of $\psi$ is $2^{r}$.

From Lemmas 1 and 2 we get

THEOREM 1. If a compact Lie group $G$ is also an $H$-cogroup, then $\operatorname{rank} G=1$.

COROLlaRY. If $n \geqq 1$ and $S^{n}$ can be made into a topological group, then $S^{n}$ is a Lie group of rank 1 .

Proof. A group manifold is a Lie group, and $S^{n}$ is compact, so Theorem 1 applies.

Theorem 2 (Following Samelson). If $G$ is a compact lie group of rank 1 , and $\operatorname{dim} G=n>1$, then $n=3$.

Proof. Since $G$ is compact we can get an inner product on its Lie algebra $g$ such that the adjoint representation

$$
\text { Ad: } G \rightarrow G L(\mathrm{~g})
$$

is such that each $\operatorname{Ad}(g)$ is orthogonal; i.e., $\operatorname{Ad}(G) \subset O(g)$ the orthogonal group on $g$.

Let $T=S^{1}$ be a maximal torus of $G$ and let $X$ be a unit vector in $g$ tangent to $T$. Define

$$
\phi^{\prime}: G \rightarrow S^{n-1} \subset_{g}
$$

by $\phi^{\prime}(g)=\operatorname{Ad} g X$.

LEMMA. $\phi^{\prime}$ induces a homeomorphism $\phi: G / T \rightarrow S^{n-1}$.

Proof of Lemma. First off, $\phi^{\prime}$ does induce $\phi$ since if $t \in T$

$$
\phi^{\prime}(g t)=\operatorname{Ad}(g t) X=\operatorname{Ad}(g) \operatorname{Ad}(t) X=\operatorname{Ad}(g) X=\phi^{\prime}(g) .
$$

Second, $\phi$ is injective for if

$$
\operatorname{Ad} g X=\operatorname{Ad} h X,
$$

then $\operatorname{Ad}\left(g h^{-1}\right) X=X$. Thus $\operatorname{Ad}\left(g h^{-1}\right)$ leaves the one-dimensional Lie algebra $\mathcal{L}(T)$ of $T$ elementwise fixed. Since $T$ is maximal this implies $g h^{-1} \in T$.

Finally, $G / T$ is a compact closed $n-1$ manifold and it follows that $\phi$ is surjective, so the Lemma is proved. 
Since $\phi$ maps $G / T$ onto $S^{n-1}$ it follows that there exists $g_{0} \in G$ such that Ad $g_{0} X=-X$. This allows us to get a map $f: S^{2} \rightarrow S^{n-1}$ as follows. Let $\omega:[0,1] \rightarrow G$ be a path from the identity $e$ to $g_{0}$. Then since $T=S^{1}$ we have a map

$$
F: S^{1} \times I \rightarrow G
$$

given by $F(t, \tau)=\omega(\tau) t \omega(\tau)^{-1}$. Then $F\left(S^{1} \times 0\right) \subset S^{1}$ and $F\left(S^{1} \times 1\right) \subset S^{1}$ so $F$ induces a map $f: S^{2} \rightarrow G / S^{1}=S^{n-1}$. We assert that $f$ is essential.

If $f$ is nullhomotopic, we can cover a nullhomotopy in $G$ to get a homotopy

$$
\Phi: T \times I \rightarrow T
$$

with $\Phi_{0}=F(-, 0)$ the identity and $\Phi_{1}=F(1,1)$ sends $t$ to $t^{-1}$. No such homotopy exists, so $f$ is essential and we conclude that $S^{n-1}=S^{2}$ and $n=3$.

THEOREM 3. The $n$-sphere $S^{n}(n>0)$ can be made into a topological group if and only if $n=1$ or $n=3$.

Proof. Use Theorem 2 and the corollary to Theorem 1.

REMARK. SO(3) is a compact Lie group of rank 1 and dimension 3, so that it could be a cogroup as far as the results above are concerned. But Hilton [1] has shown that a comultiplication on $X$ implies $X$ has Lusternik-Schnirelmann category $\leqq 2$, whereas $\mathrm{SO}(3)=P^{3}$ has category 4.

\section{REFERENCES}

1. P. J. Hilton, On a generalization of nilpotency to semi-simplicial complexes, Proc. London Math. Soc. 10 (1960), 604-622.

2. H. Hopf, Über der Rang geschlossener Liescher Gruppen, Comment. Math. Helv. 13 (1940-1941), 119-143.

3. H. Samelson, Über die Sphärer, die als Gruppenräume, auftreten, Comment. Math. Helv. 13 (1940), 144-155.

4. - Topology of Lie groups, Bull. Amer. Math. Soc. 58 (1952), 2-37.

5. E. Spanier, Algebraic topology, McGraw-Hill, New York, 1966.

RICE UNIVERISTY AND

The University of Texas 